\title{
Commentary on Hussey and Hughes (2020): Hidden invalidity among 15 commonly used measures in social and personality psychology
}

\author{
Eunike Wetzel $^{1}$ \\ Brent W. Roberts ${ }^{2}$ \\ ${ }^{1}$ Department of Psychology, Otto-von-Guericke University Magdeburg, Germany \\ ${ }^{2}$ Department of Psychology, University of Illinois at Urbana-Champaign
}

Accepted version of article in press at Advances in Methods and Practices in Psychological Science. This paper is not the copy of record and may not exactly replicate the final, authoritative version of the article.

Date of acceptance: August 16, 2020

Correspondence concerning this article should be addressed to Eunike Wetzel, Department of Psychology, Otto-von-Guericke University Magdeburg, Germany.

Email: eunike.wetzel@ovgu.de 


\title{
Commentary on Hussey and Hughes (2020): Hidden invalidity among fifteen commonly used measures in social and personality psychology
}

\begin{abstract}
Hussey and Hughes (2020) analyzed four aspects (internal consistency, test-retest reliability, factor structure, and measurement invariance) relevant to the structural validity of psychological scales in 15 self-report questionnaires and concluded that social and personality psychology has a "hidden invalidity" problem. We argue that their argument that the field ignores structural validity (hence "hidden") is incorrect because many published papers specifically investigate the measurement properties of instruments applied in social and personality psychology. Furthermore, we show that the models they used to test structural validity do not match the construct space for many of the measures. Lastly, we argue that their conclusion that measures are invalid based on a pass/fail decision for measurement invariance is overly simplistic. Rather, partial measurement invariance and the effect size of the noninvariance should be considered. Moving forward, we think it would be important for all researchers to more actively engage with prior measurement research, know the limits of existing measures, and invest in a deeper examination of the psychometric properties of their own measures in each of their studies.
\end{abstract}

Author contributions: EW and BWR wrote and revised the commentary. 
Hussey and Hughes (2020) analyzed four aspects relevant to the structural validity of a psychological scale (internal consistency, test-retest reliability, factor structure, and measurement invariance) in 15 self-report questionnaires, some of which are very popular such as the Big Five Inventory (John \& Srivastava, 1999) or the Rosenberg Self-Esteem Scale (Rosenberg, 1965). Our commentary will focus on 1) their argument that measurement issues like these are ignored, 2) that the models used to test structural validity do not match the construct space for many of the measures, and 3) the analyses and conclusions regarding measurement invariance were needlessly limited to a dichotomous decision rule.

First, we believe it is important to note that we are in agreement with the sentiment behind Hussey and Hughes (2020) and the paper that appeared to inspire their work (Flake, Pek, \& Hehman, 2017). When it comes to the top journals in personality and social psychology, measurement issues are seldom the focus of the articles published therein, nor is the quality of the measures used by researchers a top priority in evaluating the value of the research. Furthermore, the use of ad-hoc measures is common in some fields.

Nonetheless, we disagree with the authors' analyses, interpretations and conclusions concerning the validity of these 15 specific measures for three reasons. First, the authors argue that structural validity is rarely reported in the literature. Readers may conclude that the field is cavalier about the quality of their measures. While the top journals may not publish work on structural validity, hundreds of studies are published annually in measurement focused journals on exactly that topic. Thus, the situation is actually worse than that portrayed by recent criticisms. The field does not undervalue good measurement practices because the research has not been done. The field so undervalues good measurement practices that it ignores the measurement research despite the fact that it has been done. 
As a case in point, we conducted a cursory google scholar search for papers focusing on measurement invariance for each of the scales considered in Hussey and Hughes (2020) ${ }^{1}$. We collated papers reporting on the structural validity of the specific and related scales reported in Hussay and Hughes (2019) $)^{2}$. As Table 1 in the online supplement shows, we found, in most cases, multiple papers testing the structural validity of each measure.

Moreover, in several cases there is a robust, decades long lineage of research showing that not only do scholars care about measurement issues, but that these issues have been and continue to be the obsession of many researchers and many research reports. Leaving readers with the impression that the field does not care about or publish research on these measurement issues would be a disservice to the many researchers, disproportionately in applied fields like Industrial/Organizational Psychology, that do wrestle with these issues on a continuous basis.

The second issue that we would like to raise is the danger of unexamined assumptions and the application of potentially inappropriate standards when they are not warranted. The authors use confirmatory factor analysis (CFA) models with several assumptions built in presumably because they are considered the best and most appropriate for evaluating the measures in their study. There are at least two reasons not to make this assumption.

First, the standards used in CFA models, while accepted by the methodologists that employ them, were not established to develop and evaluate new measures. These standards are arbitrary in their own right (see below and Hopwood \& Donnellan, 2010). Second, the authors chose to assume that constructs should have items with no-cross loadings within the CFA measurement models. This assumption fails to consider the theoretical construct space being tested in the case of several measures, especially personality traits. Specifically, the

\footnotetext{
${ }^{1}$ osf.io/msv2f

We conducted a Google Scholar search with "measurement invariance" combined with the specific scales reported in Hussey and Hughes (2020) and limited the search to the first page. ${ }^{2}$ In the case of some scales, the specific scale had not been the focus of prior work, but closely related scales had been. In those cases, we listed the closely related work.
} 
assumption that factors should have no cross-loadings contravenes what we know about the items that go into personality trait inventories. If one examines the history of measurement research on the Big Five one finds that almost all Big Five personality items are multifactorial (Hofstee, De Raad \& Goldberg, 1992). For example, "dependable" and "reliable" load on both agreeableness and conscientiousness. On average, most terms we use to describe people load on two factors not one. Thus, the seemingly reasonable practice in CFA modeling that calls for no cross-loadings for any factors is inconsistent with the construct space of personality inventory items. The choice to value and prioritize no-cross loadings may be something to aspire to in some measurement spaces. Nonetheless, it does not match the factorial complexity represented in the content of most personality items, and most likely most of the items used for all of the measures considered in Hussey and Hughes. Without the knowledge of the history of attempts to use CFA with the Big Five (Vassend \& Skrondal, 1997) and without the knowledge of the factorial complexity of the Big Five, this seemingly innocuous decision was problematic from the start. If the goal is to test the structural validity of these measures, ideal modeling practices should be driven by theoretical and conceptual understanding of the construct space, and not what may be common, but less optimal default methodological practices.

Finally, the authors accurately describe the evaluative systems for determining measurement invariance across groups as unsettled, but then go on to portray the field as if there is one accepted threshold and ignore several solutions that have helped applied researchers for years to evaluate the importance of putative differences in measurement invariance (MI) models. Hussey and Hughes (2020) use a "two-metric strategy" to evaluate MI across groups such that .01 changes in CFI and .015 changes RMSEA were the thresholds used to judge whether a scale demonstrated configural, metric, or scalar invariance. These thresholds were the only standards provided and used for qualitative distinctions such as "failing" to establish measurement invariance. They conclude that MI was poor for 14 out of 
15 questionnaires (or 25 out of 26 subscales) and that global structural validity is therefore questionable, implying that researchers should stop administering these scales.

There are a number of problems with their analyses and in particular their conclusion which we highlight in the following: 1) MI is not a pass/fail dichotomy, rather it is a matter of degree. 2) Partial MI allows drawing comparisons across groups. 3) There are many methods of testing MI, some of which also take the effect size of the noninvariance into account.

Hussey and Hughes (2020) imply that when the fit criteria are not met, measures fail structural validity and therefore should not be used to make comparisons across groups. This is too simplistic. The procedure they applied for testing MI is a global test of whether full MI exists across all items in a scale. However, it is possible that the majority of items are invariant and only one or a few items are noninvariant. In this case, partial MI might still be achieved. All that is necessary for partial MI is for some items to be invariant, not all of them. The invariant items establish a common metric across groups, allowing comparisons to be drawn in the final partial MI model (Byrne, Shavelson, \& Muthén, 1989; Reise, Widaman, \& Pugh, 1993; Schmitt \& Kuljanin, 2008; Steenkamp \& Baumgartner, 1998). When there are few noninvariant items relative to the number of invariant items, estimates of the mean differences across groups will be unbiased (Guenole \& Brown, 2014). Thus, with partial MI, it is still possible to compare groups, although this requires the estimation of latent mean differences because that is the only way to control for the noninvariance. Mean differences computed from observed scores, on the other hand, will be biased.

Hussey and Hughes (2020) used one particular method of testing MI that is based on comparing model fit to cut-off criteria. It should be noted that there are many other methods for testing MI that might lead to different results such as non-parametric methods and methods in the framework of item response theory (IRT), where MI is referred to as differential item/test functioning (for an overview see Penfield \& Camilli, 2007 and for a comparison between CFA and IRT approaches see Tay, Meade, \& Cao, 2015). IRT-based 
methods usually take a different approach than CFA methods that relies on considering the effect size of the noninvariance (for an overview see DeMars, 2011), such as the classification system developed by Educational Testing Service (Zieky, 1993), although effect size methods have also been developed for CFA (Nye \& Drasgow, 2011). The advantage of these methods is that only substantial (e.g., moderate or large) noninvariance is flagged whereas with other methods, especially those that are sample-size dependent (significance testing, delta CFI), even noninvariance of negligible size might lead to the rejection of MI. Thus, the conclusion that 15 out of 16 measures have questionable structural validity because they have poor MI in one study using one criterion is unwarranted. Moving forward, more rigorous and nuanced investigations of the measurement invariance of popular personality measures are needed. Ideally, checking the items for invariance across commonly formed groups (e.g., gender, age) would already be part of the test construction process, as in educational testing.

Taken at face value, the arguments by Hussey and Hughes (2020) and others leave one with the impression that there is a crisis in psychological measurement in the field of social and personality psychology caused by either poor measurement or a lack of good measurement research. In our opinion, the real problem is that psychological measurement is difficult, complex, and requires more effort and energy than most researchers are willing to invest. Consumers of psychological measurement, arguably, want nothing more than to pull measures off the metaphorical shelf. This is unwise. We believe a more productive way forward is for all researchers to more actively engage with prior measurement research, know the limits of existing measures, and invest in a deeper examination of the psychometric properties of their own measures in each of their studies. This can involve using measurement models that conform to the theoretical construct space by allowing cross-loadings, only using observed scores when it is justified, and considering effect sizes and partial invariance in measurement invariance analyses. We believe a more measured approach to measurement will improve all research efforts. 


\section{References}

Byrne, B. M., Shavelson, R. J., \& Muthén, B. (1989). Testing for the equivalence of factor covariance and mean structures: The issue of partial measurement invariance. Psychological Bulletin, 105(3), 456-466. doi:10.1037/0033-2909.105.3.456

Chen, F. F. (2007). Sensitivity of goodness of fit indexes to lack of measurement invariance. Structural Equation Modeling-a Multidisciplinary Journal, 14(3), 464-504.

DeMars, C. E. (2011). An analytic comparison of effect sizes for differential item functioning. Applied Measurement in Education, 24(3), 189-209.

Flake, J. K., Pek, J., \& Hehman, E. (2017). Construct validation in social and personality research: Current practice and recommendations. Social Psychological and Personality Science, 8 (4), 370-378.

Guenole, N., \& Brown, A. (2014). The consequences of ignoring measurement invariance for path coefficients in structural equation models. Frontiers in Psychology, 5, 980. doi:10.3389/fpsyg.2014.00980

Hofstee, W. K., De Raad, B., \& Goldberg, L. R. (1992). Integration of the Big Five and circumplex approaches to trait structure. Journal of Personality and Social Psychology, 63(1), 146.

Hopwood, C. J., \& Donnellan, M. B. (2010). How should the internal structure of personality inventories be evaluated? Personality and Social Psychology Review, 14(3), 332-346. doi:10.1177/1088868310361240

Hu, L. T., \& Bentler, P. M. (1999). Cutoff criteria for fit indexes in covariance structure analysis: Conventional criteria versus new alternatives. Structural Equation Modeling, 6(1), 1-55. doi:10.1080/10705519909540118

Hussey, I., \& Hughes, S. (2020). Hidden invalidity among 15 commonly used measures in Social and Personality Psychology. Advances in Methods and Practices in Psychological Science, 3(2), 166-184. doi:10.1177/2515245919882903 
John, O. P., \& Srivastava, S. (1999). The Big Five trait taxonomy: History, measurement, and theoretical perspectives. In L. A. Pervin \& O. P. John (Eds.), Handbook of personality theory and research (pp. 102-138). New York: Guilford Press.

Nye, C. D., \& Drasgow, F. (2011). Effect size indices for analyses of measurement equivalence: understanding the practical importance of differences between groups. Journal of Applied Psychology, 96(5), 966-980. doi:10.1037/a0022955

Penfield, R. D., \& Camilli, G. (2007). Differential item functioning and item bias. In C. R. Rao \& S. Sinharay (Eds.), Handbook of statistics (Vol. 26, pp. 125-167). Amsterdam, The Netherlands: North-Holland.

Reise, S. P., Widaman, K. F., \& Pugh, R. H. (1993). Confirmatory factor analysis and item response theory: Two approaches for exploring measurement invariance. Psychological Bulletin, 114(3), 552-566.

Rosenberg, M. (1965). Society and the adolescent self-image. Princeton, NJ: Princeton Universiy Press.

Schmitt, N., \& Kuljanin, G. (2008). Measurement invariance: Review of practice and implications. Human Resource Management Review, 18(4), 210-222. doi:10.1016/j.hrmr.2008.03.003

Steenkamp, J. B. E. M., \& Baumgartner, H. (1998). Assessing measurement invariance in cross-national consumer research. Journal of Consumer Research, 25(1), 78-90. doi:10.1086/209528

Tay, L., Meade, A. W., \& Cao, M. Y. (2015). An overview and practical guide to IRT measurement equivalence analysis. Organizational Research Methods, 18(1), 3-46. doi:10.1177/1094428114553062

Vassend, O., \& Skrondal, A. (1997). Validation of the NEO Personality Inventory and the five-factor model. Can findings from exploratory and confirmatory factor analysis be reconciled?. European Journal of Personality, 11(2), 147-166. 
Zieky, M. (1993). Practical questions in the use of DIF statistics in item development. In P. W. Holland \& H. Wainer (Eds.), Differential item functioning (pp. 337-347).

Hillsdale, NJ: Lawrence Erlbaum. 